\author{
Military Technical College \\ Kobry El-Kobbah, \\ Cairo, Egypt
}

14th International Conference on

Applied Mechanics and

Mechanical Engineering.

\title{
Sensing Strategy for Multi-Arm Mobile Robot Navigation For Nuclear Decommissioning Tasks
}

\author{
Khaled M. Zied ${ }^{*}$ and Mohamed J. Bakari**
}

\begin{abstract}
:
The primary use of robotics in nuclear decommissioning applications is to reduce the radioactive dose levels to which workers are exposed during the decommissioning processes. There are many situations where, owing to the degree of radiation and the very long half-lives of the radioactive materials involved, robotics is the only feasible option. However, unstructured decommissioning environments pose significant challenges to the navigation, operation and control of tele-operated mobile robots and more specifically multi-arm mobile robots. Through task planning is often not achievable due to the risk of radiation and the unpredictable nature of the decommissioning workspace environment. The hazardous and unpredictability of the decommissioning environment makes real-time sensing with vision system and decision making an essential component of the multi-arm mobile robot. This paper proposes the use of a sensing strategy and visual feedback from cameras for navigation and position estimation and to provide the operator with real-time information of the target and its surrounding for controlling the multi-arm mobile robot and the successful execution of the decommissioning task.
\end{abstract}

\section{Keywords:}

Nuclear decommissioning, Multi-arm robot, robot controller, sensing strategy

\footnotetext{
${ }^{*}$ Mechanical Design Department, Faculty of Engineering, Mataria, Cairo 11718 kmzied@hotmail.com
} 
Proceeding of the $14^{\text {th }}$ AMME Conference, 25 -27 May 2010

** LPDU, Engineering Department, Lancaster University, Lancaster, UK, LA1 4YR, m.bakari@lancaster.ac.uk 


\section{Introduction:}

Much of the decommissioning process utilizes well established demolition techniques although there are complications within this process due to the risk of exposure of workers to radiation. Current technologies are often labour intensive, time consuming, expensive, or unnecessarily expose workers to the hazardous material. In the nuclear industry, robotic systems are usually required in nuclear decommissioning environments that are too hazardous for direct human involvement. Sophisticated intelligent control systems are currently insufficiently developed to allow these robots to operate under their own initiative; therefore any sophisticated decommissioning tasks still need human operator involvement.

The nuclear industry, however, does currently use tele-robotics for purposes such as site inspection and other decommissioning tasks.

Robotic systems provide a potential solution to decommissioning problems because the robots can be remotely controlled from an operator console and provide visual feedback to the operator while executing the decommissioning tasks; thus reducing the radioactive dose levels to which workers are exposed. However, current automated systems employ virtually no autonomy or even programmed motion. Invariably there is a human in the control loop, and this is expected to continue. This means that nearly all systems employ simply remote control, tele-operation or master/slave manipulation. Systems generally fall into one of four categories:

1. Relatively expensive customised solutions to specific problems

2. General purpose plant

3. Systems fabricated from off-the-shelf components

4. Automated process plant for packaging and waste processing.

It has been noted by researchers and decommissioning agencies around the world the advantages of using multi-arm robots over single-arm robots Alford \& Belyeu [1], Cox et al [2] and Miyabe et al [3]. A multi-arm robot system has the ability to perform two distinct operations simultaneously or separately, it also has the ability to perform the same processing operation in a coordinated manner, or share a task such as unscrewing and dismantling two components apart or holding a cutting a pipe. Previous researches concerning multi-arm robot systems have been deployed by either a rigid boom overhead transporter or by a crane rather than a remote vehicle.

Figure (1): MARS-ND System

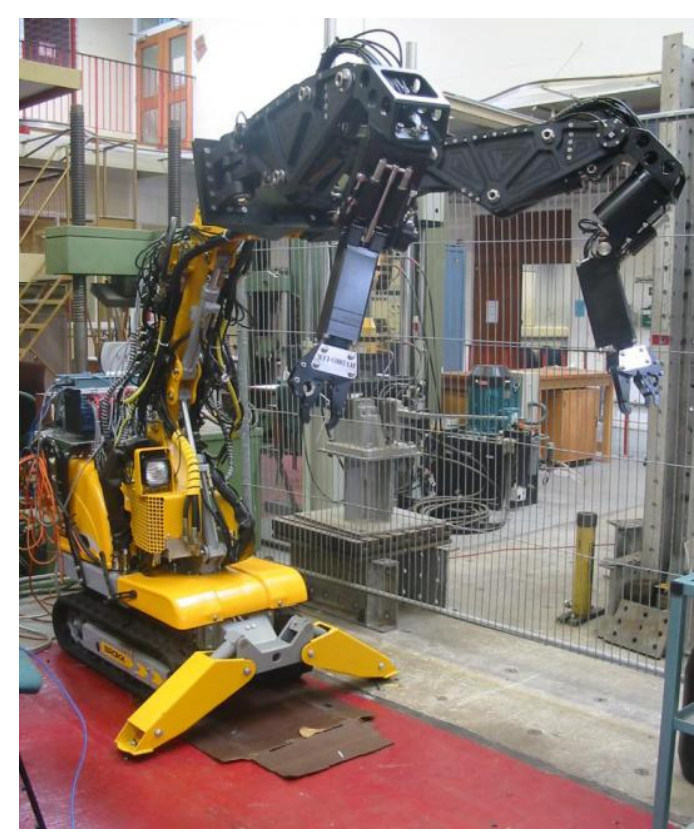

Examples of these systems are dual-arm work platform (DAWM) Noakes [4], cranedeployed work platform RODDIN Desbats [5], Advanced Servo-Manipulator (ASM) \& Dual Arm Mobile Working Platform (PTM) Parker and Draper [6] and Two-Arm Bilateral Servo Manipulator System (BSM) Nakamura [7]. Both systems are multi-arm robot platform 
equipped with two manipulators and mounted to a base and transported by cranes. Neither of these two system manipulators are light weight or small enough to be manoeuvred into small and restricted areas.

The utilization of sensors is considered on three areas:

1. Surveillance and monitoring of the working area

2. Global localisation of robotic systems

3. Fine positioning of the end-effectors of the system

Vision systems are an essential component of a robotic system and they find many applications such as environmental mapping and the system control. For example, Macphater [8] reported a complete remote measuring system for mapping of hazardous environments called HAZMAP which comprises a sophisticated vision system that allows operators to monitor the working areas remotely. This allows the building of a 3D CAD model and this system has been tried successfully in several hazardous environments including nuclear power stations.

Woodfill et al [9] presented a vision system for real time vision for mobile robot control in unstructured environments. This system is designed to pick up moving objects in the scene. Although this work is not suitable for environment mapping, it is very useful in controlling the motion of the end-effector of a robot using vision system.

Bevan et al [10] reported a sensory system comprising a vision system that is used for controlling a climbing robot, ROBUG I, using a high level command controller. This vision system consists of multiple video cameras positioned at different locations. Two ultrasonic sensors and clinometers are used for obstacle avoidance in unstructured environments while the climbing robot is in transition from floor to wall positions. The application area for this robot is the inspection of hazardous unstructured environments.

Laser range finders find wide application in outdoor applications such as localisation and safety field violation monitoring. Tonaki and Takada [11] presented a positioning system comprises two laser scanners used for position estimation of a moving target such as machinery or a robotic system relative to a pre-determined position. A GPS system was employed to verify the correctness of the estimated position obtained using the laser scanner. Accurate results were obtained from the new system.

In the present work, as part of the global development process of the MARS-ND robot Figure 1, a sensory system is proposed to satisfy the user and system requirements specified and reported previously in [12]. One of the basic functions of MARS-ND multiarm robot is to position tools in the appropriate position to perform the predetermined tasks. This function is easy to satisfy once the robot itself is positioned in a pre-determined position. To achieve this objective a sensing strategy is prepared, based on available technology such as laser and ultrasonic technologies. The sensing strategy for MARS-ND includes robot base positioning, task area monitoring, the end-effector positioning and the end-effector alignment. The present work is limited to the identification and development of only part of the system. Section 3 identifies the part of the development process.

\section{MARS-ND Robotic System}

The off-the-shelf components used to develop the MARS-ND as shown in Figure 1 consist of the smallest Brokk demolition machine, the Brokk 40, as a mobile platform; and two Hydro-Lek robot manipulators as shown in Figure 1. The Brokk 40 machine consists of a 
moving vehicle with a single five degree of freedom manipulator, hydraulic tank, controller and remote-control device. The multi-arm system attached to the Brokk platform is HydroLek HLK-7W with continuous jaw rotation mechanism. This consists of two seven-function manipulators (six rotary joints with a gripper). A system of wireless communication has been developed to control the manipulators and obtain feedback.

The Multi-Arm Mobile Robot System was developed following the refined design and system development process for nuclear decommissioning application. This development process has already been applied to the development of Starlifter construction robot Zied, K. [13] See Figure 2. It can also be adapted and applied to other research fields including space robot applications and subsea.

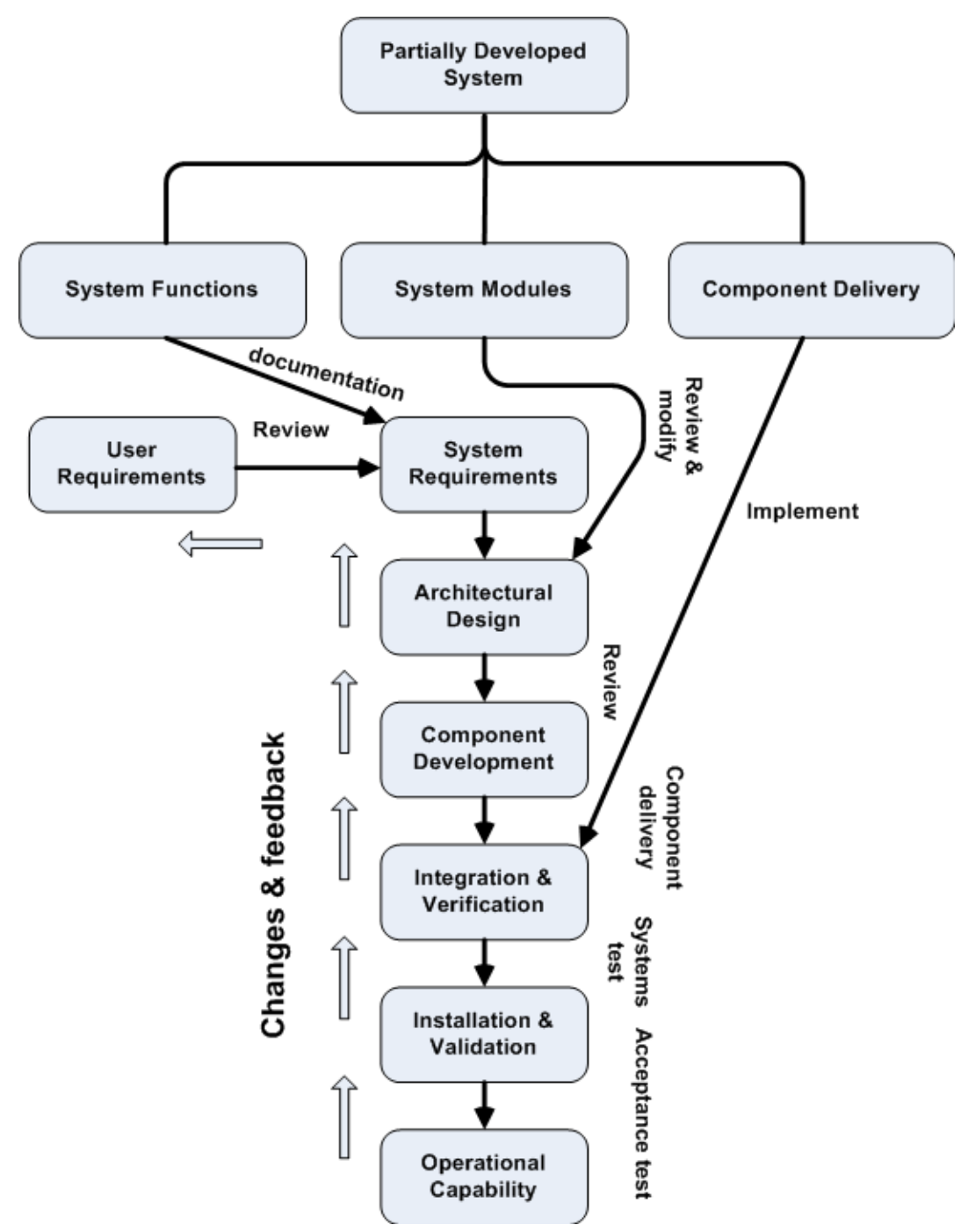

Figure (2): System development process for MARS-ND

\section{a. Hardware and Software Integration}

A system integration process was carried out physically by bringing together many subsystems and their components into one system and ensuring that the subsystems fit and function together as one system. Figure 3a shows a layout of hardware integration where two Hydro-Lek arms are mounted on to a mounting bracket and the distance between the two arm bases is $500 \mathrm{~mm}$. The mounting bracket was designed to hold the weight of the arms with full payloads and also to act as a stable platform for the two arms. 
The other side of the bracket is designed to be fitted to the end of the Brokk machine manipulator where it can be rotated forward and backward.

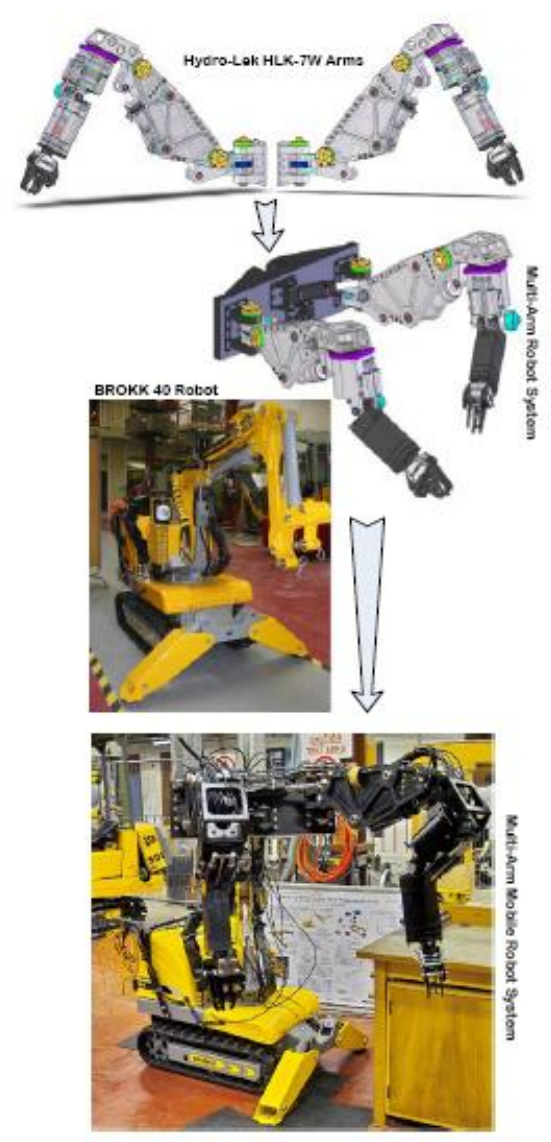

(a)

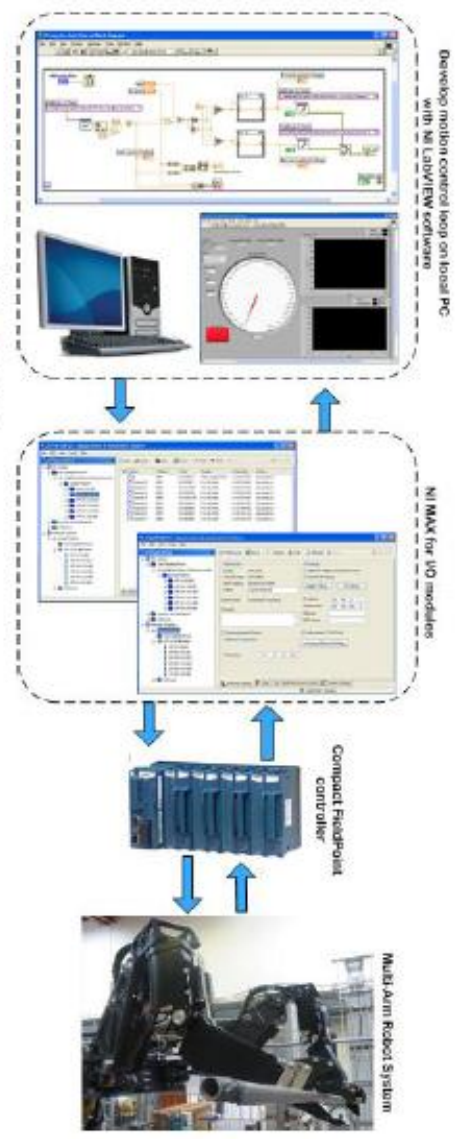

(b)

Figure (3): Hardware and Software Integration
(a) Hardware Integration
(b) Software Integration

Developing software for controlling robotic systems is expensive due to the inherent complexity of these systems. Therefore, there is a need for tools that permit a reduction in the programming efforts, aiming at the generation of modular and robust applications. LabVIEW software is an object orientated framework for programming robotic systems and provides a full-featured graphical programming tool for developing measurement, automation and control applications. At the current stage of the research, some task performance has been carried out for simple pipe cutting and manipulation tasks using the Multi-Arm robot system. This has been undertaken in order to examine the feasibility of the motion control applications created for the control of the robot joints and the feedback from the sensors.

\section{Sensing strategy for MARS-ND}

The proposed sensory system for the MARS-ND robot is identified according to the available sensors. The sensory system functions identified for MARS-ND are:

1. Robot base positioning.

2. End effectors alignment.

3. Task area monitoring. 
The functional decomposition of the sensory system proposed here is shown in Figure (4). The work presented here is limited to the first objective outlined above. Most of the work done here is conducted in order to achieve the following objectives:

1. Interface with the sensors

2. Sensor data acquisition, processing and visualization

3. Examination of the performance of the sensors for indoor and outdoor environments

4. Validation of the sensor information

5. Sensing strategy planning

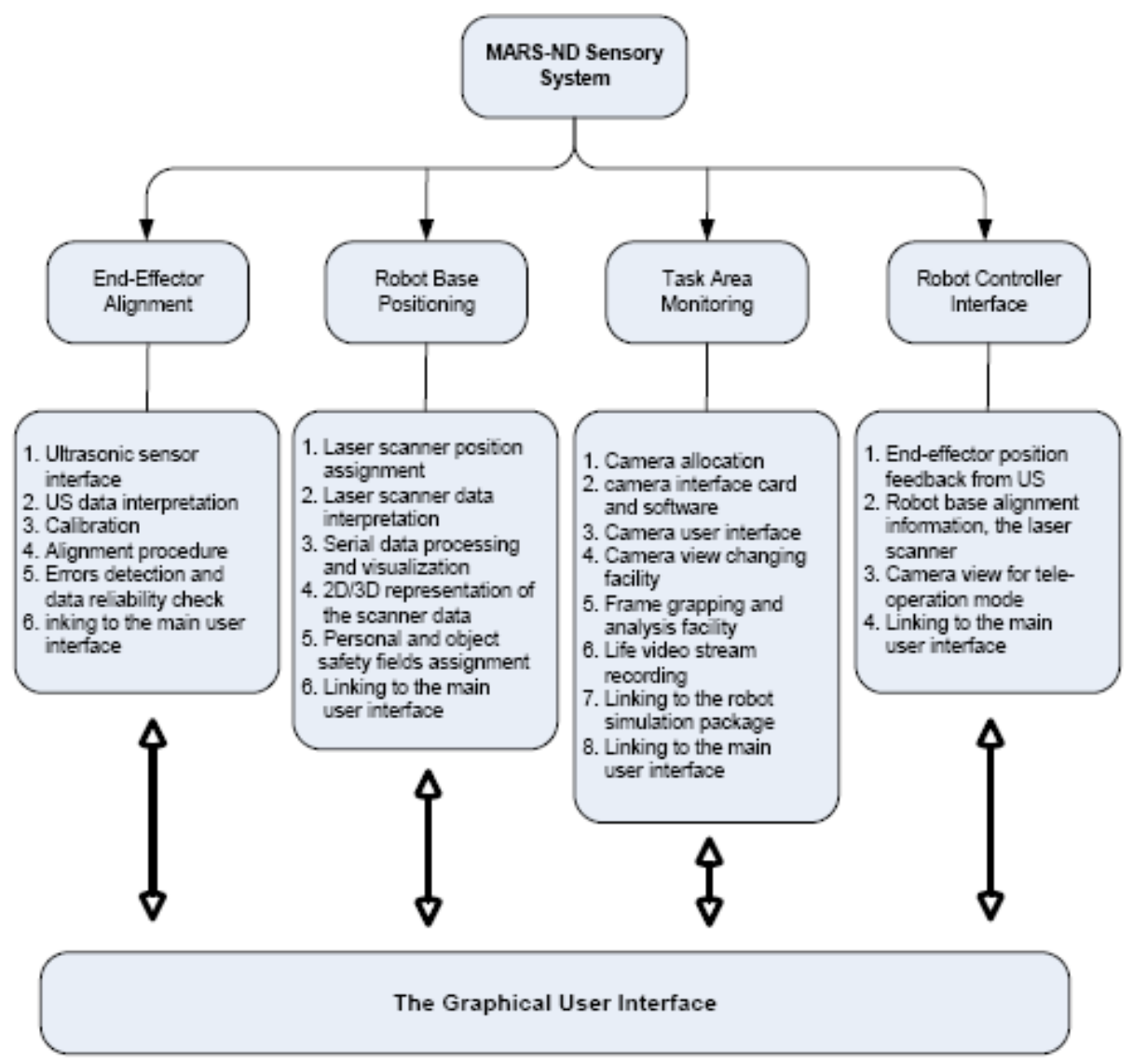

Figure (4): Functional decomposition of the MARS-ND sensory system

The three objectives outlined above for the sensory system employed in the current system can be achieved using the following sensors:

\section{a. A laser scanner}

A laser scanner is attached to the Brokk robot base to scan the area in front of the robot to assist the operator in positioning the robot in a suitable place relative to the working area. During the navigation of the robot base the two arms are positioned in a pre-determined home position to ensure safety of the two arms. The area in front of the robot detected by the laser scanner should accommodate the two arms when they are in the home position and the operational position of the two arms. The laser scanner can move up and down in some stations in the navigation path which will be determined using the vision system, to 
check for front and side obstacles at different levels to give three dimensional map of the navigation area.

The RotoScan laser scanner -see Figure 6, is a two dimensional range sensor that can detect objects of size of $70 \mathrm{~mm}$ in a semi circle with radius up to $25 \mathrm{~m}$. It can be considered as an optical, area radar unit. The main application for this unit is in safety related applications for object and personnel detection such as driverless transportation and safeguarding dangerous machine areas. The Leuze laser scanner can be used for object contour detection for manufacturing applications. The unit has the facility to adjust the safety fields at which objects and personnel can be detected. A user interface has been developed for the laser scanner that provides data interpretation, data processing and 2D visualisation. A 3D map can be constructed by scanning the task area at different planes. Each plane's data can be stored and plotted against the position of the laser scanner using a position sensor attached to the laser scanner. The aim of using the laser scanner, in combination with a camera view, is to protect the robot and the working environment from collision during robot positioning as demonstrated in Figure 5. The robot can operate correctly within its working envelope. The camera view gives a 2D image of the working area and the laser scanner gives the third dimension.

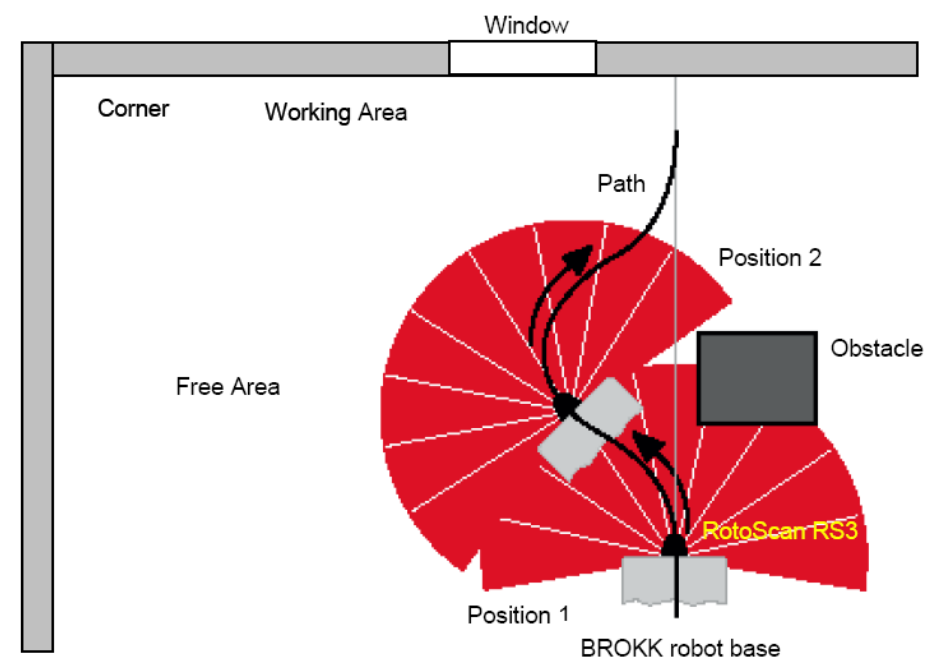

Figure (5): The laser scanner sensing strategy

Several tests have been carried out previously on the laser scanner on a similar mobile platform [13]. From these tests several conclusions have been withdrawn regarding the use of the laser scanner in construction applications particularly in collision avoidance and safety related applications. These conclusions are summarized as follows:

1. The reliability and accuracy of the readings of the laser scanner degrade mostly with the increased vibration induced during the motion of the sensor on rough terrain.

2. Accurate measurements can be obtained when the robotic platform is stationary and close to the targeted objects (obstacles)

3. The laser scanner seems to be ideal for detection of flat uniform surfaces perpendicular to the scanning plane. Irregular surfaces are detectable but cannot be identified through the readings of the scanner because of the disturbances induced by the irregularity of the surfaces.

4. Reflectivity of the detected surfaces has a major effect of the reliability of the scanner readings. For example, glass or water surfaces are not detectable to any 
extent and they result mostly in spurious readings. This coincides with the scanner characteristics identified by the manufacturer regarding reflectivity of detected surfaces as shown in Figure (6).

Despite the limitations outlined above, the laser scanner performs well particularly for rough estimations of distances relative to the robot base as is intended in the present work.

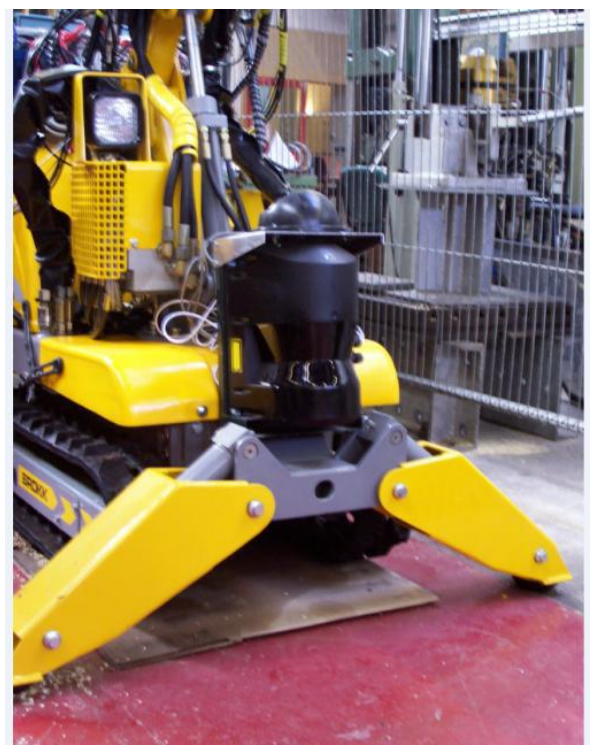

(a)

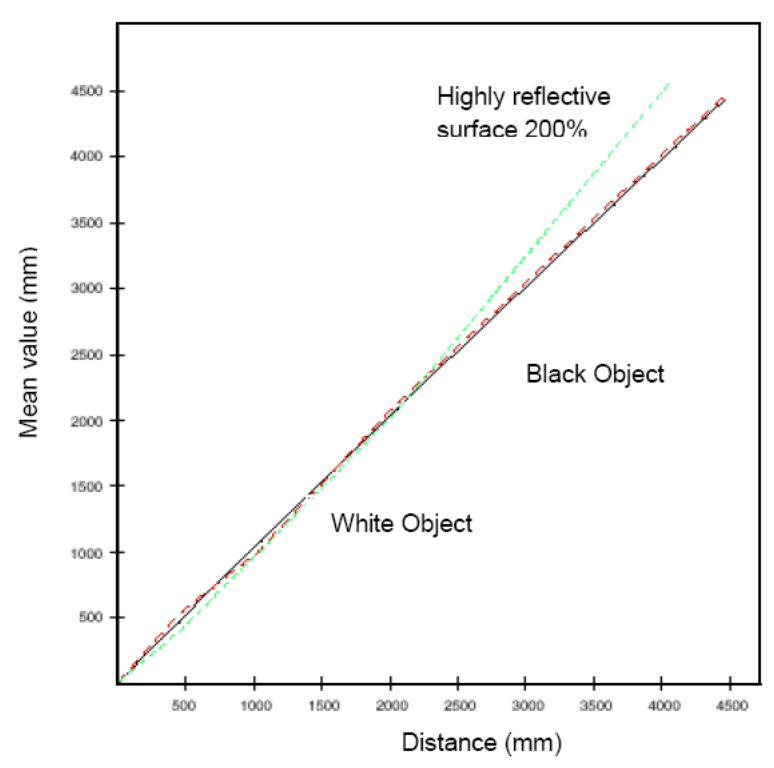

(b)

Figure (6): (a) The laser scanner on the Brokk (b) Sensitivity of the laser scanner to variation of reflectivity of surfaces [13]

\section{b. Ultrasonic sensors}

The working area should be within the working envelope of the two arms so that the arms can perform the task successfully. A collision avoidance algorithm is under development for the two arms to ensure safe operation of the two arms together. The work is underway to employ a sensory system to achieve this objective. A set of ultrasonic sensors are proposed to be used for the alignment of the end effectors relative to the task area.

\section{c. Video camera system}

Task area monitoring is an essential function of the sensory system for MARS-ND. A vision system is attached to the system using multiple cameras to give full coverage of the navigation path and the working area after placing the robot in the required position. The tele-operation facility in the system mainly depends on vision system as well as the laser scanner to place the robot in place and the task performance. Task monitoring by the system operator requires the use of video cameras placed at different locations on the system. The use of video cameras could be for monitoring only or be useful in extracting environment features by using simple image processing facilities such as dimensioning of the work area. Video camera systems are available as off-the-shelf components however; 
the selection of a suitable camera for decommissioning activity monitoring should consider the special nature of the working environment.

In addition to other factors related to the nature of the working environment which requires a durable and reliable camera system. In the current work multiple video cameras are connected to a video card to develop a monitoring system integrated with the sensory system discussed above. A stand alone user interface has been developed within LabVIEW to interface with the video card. This user interface is based on the use of ActiveX components which includes several functions for video recording, frame grabbing in addition to image file manipulation functions. Image acquisition functions (IMAQ) built in LabVIEW are used for frame capturing, saving, and simple image processing such as zoom-in and zoom-out. The use of these functions can be extended to add other facilities such as on-screen measurements to extract dimensions and features of the working area.

\section{Conclusion}

The current work reports the latest development of the MARS-ND robotics system for nuclear decommissioning tasks. As explained the primary use of robotics in nuclear decommissioning applications is to reduce the radioactive dose levels to which workers are exposed during the decommissioning processes. Tele-operation control strategy is used in operating the system, which requires the employment of a sensory system to assist the operator to perform the task safely and successfully. A proposal for a sensory system is presented to achieve three targets namely, positioning of the robot base relative to the working area, robot end effectors positioning and alignment and task area monitoring during task carrying out. Laser scanner and a vision system are used to achieve the first and the third task. The second task is still under investigation. Details of the performance characteristics of the laser scanner are reported to show the reliability of the system. Commercial vision system is used and integrated with the system using LabVIEwW programming environment. So far the proposed sensing strategy shows success in performing the simulated decommissioning tasks.

\section{References}

[1] Alford, C. O., and Belyeu, S. M., (1984). "Coordinate control of two robot arms". Proceedings of IEEE International Conference of Robotics, pp. 468-473.

[2] Cox, J. D., Rackers, K., and Tesar, D., (1995). "Cooperative Manipulation Experiments Using a Dual-Arm Robot". IEEE International Conference on Industrial Electronics, Control, and Instrumentation, pp. 104-109, Orlando, USA.

[3] Miyabe, T., Konno, A., Uchiyama, M., and Yamano, M., (2004). "An Approach toward an Automated Object Retrieval Operation with a Two-Arm Flexible Manipulator". The International Journal of Robotics Research, vol. 23, No. 3, pp. 275-291.

[4] Noakes, M, W., 1998. "Remote Dismantlement Tasks for the CP5 Reactor: Implementation, Operation, and Lessons Learned". Spectrum 98 International Conference on Decommissioning and Decontamination and on Nuclear Hazardous Waste Management, Denver, USA.

[5] Desbats, P., 2005. "Status and trends of remote technology applied to spent fuel management in France". CEA-CEREM/Tele-operation \& Robotics Department. IAEA-TECDOC-1433 'Remote technology applications in spent fuel management'. International Atomic Energy Agency, ISBN: 92-0-101405-8.

[6] Parker, L. E., and Drapper, J. V., 1998. Robotics Application in Maintenance and Repair, $2^{\text {nd }}$ ed. New York: Wiley. 
[7] Nakamura, H., 2005. "The state of Japanese remote technology in nuclear fuel cycle". Japan Nuclear Cycle Development Institute. IAEA-TECDOC-1433 'Remote technology applications in spent fuel management'. International Atomic Energy Agency, ISBN: 92-0-101405-8.

[8] Mcphater, N, S., 1993. "Remote measuring system for the mapping of hazardous environments". The International Symposium on Automation and Robotics in Construction (ISARC 10), pp. 333-339, Houston, USA.

[9] Woodfill, J., Zabih, R., and Khatib, O., 1996. "Real time motion vision for robot control in unstructured environments". Robotic for Challenging Environments. Prentice Hall, London.

[10] Bevan, N., Collie, A., Luk, B., and White, T., 1993. "A sensor based autonomous climbing vehicle for unstructured environments". The International Symposium on Automation and Robotics in Construction (ISARC 10), pp. 205-212, Houston, USA.

[11] Tonaki, S., and Takada, T., 1996. "Communication system of real-time two dimensional position data with scanning laser beam". The International Symposium on Automation and Robotics in Construction (ISARC 13), pp. 555-560, Tokyo, Japan.

[12] Bakari, MJ, Zied, KM, Seward, DW, 2007. "Development of a Multi-Arm Mobile Robot for Nuclear Decommissioning Tasks" International Journal of Advanced Robotic Systems, V 4 No 4 pp 502-524.

[13] Zied, K, 2004. Investigation of tools and processes for the rapid development of intelligent robotic systems for hazardous environments, A PhD Thesis, Lancaster University. 